\title{
Current possibilities of peri-operative evaluation of the radicality of primary tumour resection in breast cancer patients treated with BCT (breast conserving treatment)
}

\author{
Tomasz Nowikiewicz ${ }^{1,2}$, Wojciech Zegarski ${ }^{1,2}$
}

In accordance with the current standards of treatment of breast cancer patients, the application of breast conserving treatment $(\mathrm{BCT})$ requires a radical resection of the tumour and post-operative radiotherapy. This means the necessity of obtaining tumour-free primary resection margins, i.e. without the presence of cancer tissue. In spite of the correct pre-operative diagnostic process and appropriate surgical treatment, in about $20-30 \%$ BCT cases, the tumour resection turns out to be not radical. The necessity of performing another surgery leads to the prolongation of the overall operative time and increases costs.

A histopathological evaluation of the margin of the primary tumour resection performed on an immediate basis is not a standard procedure in patients undergoing BCT. In spite of the definite limitations of this method, its application may decrease the overall rate of revision surgeries. The paper discusses some other currently available possibilities of perioperative evaluation of the radicality of primary tumour resection in breast cancer patients treated with BCT.

NOWOTWORY J Oncol 2017; 67, 5: 285-291

Key words: breast cancer, breast conserving treatment (BCT), surgical margins, perioperative evaluation, gross clinical evaluation, microscopic evaluation

\section{Introduction}

In accordance with the current standards of treatment of breast cancer patients, the application of breast conserving treatment $(B C T)$ requires a radical resection of the tumour and post-operative radiotherapy. This means the necessity of obtaining tumour-free ("clean") primary resection margins, i.e. without the presence of cancer tissue ("no ink on the tumour" or "no tumour on the ink" - in accordance with the definition of the National Surgical Adjuvant Breast and Bowel Project - NSABP, the recommendations of the Society of Surgical Oncology - SSO and of the American Society for Radiation Oncology - ASTRO) [1-6].

A consequence of such definition of the correct manner of tumour resection is the loss of the clinical and therapeutic significance of other terms defining the scope of the primary tumour resection, such as "close margin", "focally positive margin"). The first term ("close margin") corresponded to margin of the tissues not affected by the cancer with a width lower than $2 \mathrm{~mm}$. According to the results of the studies carried out by Bolger et al., this meant a statistically higher risk of the presence of additional tumour foci in the post-operative cavity [7]. The term "focally positive margin" meant the presence of ink on the surface of the tumour (irrespective of the type of tissue) on an area smaller than $4 \mathrm{~mm}$.

Following the Dutch Guidelines for Breast Cancer Treatment, qualification of patients for adjuvant radiotherapy (within the classical BCT), without the necessity of radicalisation of the tumour resection, was possible [8].

In spite of the correct pre-operative diagnostic process and appropriate surgical treatment, in about $20-30 \%$ BCT cases, the tumour resection turns out to be not radical [9]. This means the necessity to re-operate on such patient.

${ }^{1}$ Department of Clinical Breast Cancer and Reconstructive Surgery, Oncology Centre — Prof. Franciszek Łukaszczyk Memorial Hospital, Bydgoszcz, Poland

${ }^{2}$ Chair and Department of Surgical Oncology, Ludwik Rydygier's Collegium Medicum in Bydgoszcz, Nicolaus Copernicus University in Toruń, Poland 
In case of breast intraductal cancer in situ (carcinoma intraductale - DCIS) this rate might be higher, amounting to $31-46 \%$ [10-12]. In the case of an invasive form of cancer, co-existing with DCIS - it is 11-46\% [13-15].

Apart from the prolongation of the overall treatment period and the increase of the costs, a re-operation of a BCT patient may lead to a higher rate of complications related to the treatment [16]. Additional surgery may also contribute to the discomfort felt by the patient, including lower quality of life. An important problem also related to the necessity of re-operation may by the risk of obtaining a poorer final aesthetic treatment effect [16].

One of the possibilities of the evaluation of the radicality of cancer resection during $\mathrm{BCT}$ is a histopathological verification of the tumour resection margins, performed on an immediate basis. The most frequently used method consists of a microscopic analysis of frozen specimens. In contrast to final histopathological evaluation (on the basis of the comparison of microscopic specimens made from paraffin blocs), the perioperative verification of the condition of the surgical margins does have some limitations. They concern, first of all, a lower sensitivity of the examination (65-78\%). Moreover, such an evaluation extends the overall operative time [17-19].

In accordance with the current recommendations, a peri-operative assessment of the resection margins is not a standard course of treatment in a patient undergoing BCT. This concerns, first of all, the treatment of DCIS-type lesions, in the case of which the use of immediate pathological evaluation of the specimen should be avoided [4].

So far no generally accepted level of re-operation in patients with baseline BCT has been definitely established. In the opinion of majority of authors, the re-operation rate may be regarded as an indicator of the quality of care in breast cancer patients [14]. With regards to the multiple causes of this phenomenon, expanding the tumour resection margins does not reflect directly the efficiency of the primary surgery, seems to be prevailing [20-22].

\section{Alternative methods of the evaluation of the quality of surgical margins in patients with breast conserving therapy General data}

An immediate verification of the radicality of the BCT surgery may consist of an in vivo evaluation of the tumour cavity or the assessment of the condition of the margins of the surgical specimen. With regards to the still unresolved technical limitations, the first of the listed possibilities is usually limited to attempts undertaken within clinical trials. Hence, the latter of the methods of evaluation of the quality of care in BCT proves to be more available and more widely applied.

Apart from the perioperative pathological assessment of the primary tumour margins, made with the use of the fresh frozen specimens, there are other, alternative possibilities of determination of the radicality of the procedure. They differ from each other by the type of diagnostic method and the frequency of clinical application. These methods comprise some other types of pathological assessment, radiological (imaging), fluorescent, optical and isotopic methods. Also some other surgical procedures undertaken during the resection must be mentioned (additional margin resection and gross clinical evaluation of the specimen).

\section{Pathological assessment methods}

An alternative to a perioperative pathological evaluation of the primary tumour resection margin is "touch imprint cytology" or "touch prep". This modality may also be useful during a sentinel node biopsy in patients with a lower stage of breast cancer. This study applies the characteristic property of cancer cells to adhere to clean glass surfaces and is not observed in the case of adipose cells [17].

Unlike immediate histopathological verification, the time necessary for touch imprint cytology is significantly shorter [23]. However, this possibility of applying both diagnostic methods requires some additional skills from a pathologist. As it was seen in the studies performed by Esbony et al., in the centres with in-depth experience of perioperative cytology, this examination has high sensitivity (80-100\%) and high specificity (85-100\%) [23].

The evaluation of the radicality of the breast cancer resection may also be performed by means of macroscopic margin assessment (MMA) performed on an immediate basis. This assessment consists of determination of the distance between the lesion and the edges of the resected specimen, after previous staining of the specimen surface with an ink or other dye. Once a margin with a width below $5 \mathrm{~mm}$ is found (the measurement concerns any of the radial margins: superior, inferior, lateral and medial), the scope of the tumour excision must be immediately extended in the appropriate direction $[7,24]$. In accordance with the results of the studies, the application of MMA in patients undergoing BCT decreases the rate of re-operations in comparison with the group of patients operated without the application of this method ( $26 \%$ vs $34 \%$ ) [7].

\section{Radiological (imaging) techniques}

A large group of applications of the imaging methods for the assessment of surgical margins is an outcome of the use of radiological examination for the pre-operative diagnostics of breast cancer. This is also an effect of the consensus of opinion-forming groups of experts. In accordance with the current recommendations of the Polish Society of Oncological Surgery, concerning breast cancer treatment, "it is recommended to perform a perioperative imaging assessment (mammography/ultrasound) in order to confirm a radical resection of the lesion. A peri-operative imaging assessment is performed 
with the same method which was used for the pre-operative identification of a lesion. In the case when a perioperative assessment shows that the lesion has not been resected or has not been resected radically, it is necessary to resect the proper lesion or its missing part during the same surgery and then repeat the peri-operative imagining assessment" [4].

A mammographic verification of the margins of the resected tissue specimen has become a standard procedure in the majority of centres. It allows a surgeon to obtain, in real time, information about the radicality of the performed procedure or about the necessity to extend its scope [25-27]. There is, however, some discrepancy of views concerning the clinical value of the specimen evaluated with this method. In the opinion of Britton et al. [26] and of Edwards et al. [28], this is the most useful method of verification of the resection margins out of all radiological assessment techniques. This view is additionally confirmed by the recommendations of SSO-ASTRO, concerning patients requiring a pre-operative staining of an extensive area of micro-calcifications with the use of bracketing. According to these recommendations, in the above group of patients it is necessary to perform a mammographic assessment after the resection in order to exclude the presence of residual lesions in the breast [3, 29].

Some other authors in turn, regard the correlation between the mammographic image and the result of the pathological report as highly unsatisfactory in a large number of cases [7, 25].

Another method of imaging assessment of the radicality of the primary tumour resection in BCT is a peri-operative ultrasound (USG). This concerns, in particular, the possibility of application of high-frequency ultrasound devices. As it has been seen in numerous clinical studies, such an assessment makes it possible to verify the resection margins of pathological lesions in a credible way as well as to differentiate these lesions in an accurate way [30,31]. In accordance with the results of the works carried out in 2013-2016 at the National Cancer Institute in Bangkok, which consisted in the ultrasound assessment of surgical specimen, this examination is characterised with very high sensitivity. After the consideration of the result of the final assessment of the radicality in a histopathological examination, the correlation of both methods was confirmed in $93.6 \%$ of patients with solitary tumours. In the group of patients with multiple lesions, the rate of correct assessment of surgical margins in the ultrasound was lower, yet still it was at a satisfactory level (in 75\% of studied patients). The authors point to a very high level of radicality of breast conserving procedures performed in their centre. More than $95 \%$ of patients treated in this way did not require a secondary procedure, which was also the result of the peri-operative ultrasound assessment of the surgical margins [30]. Some other studies show, however, that a more useful method for this purpose is verification with the use of a peri-operative mammography [28].
Thanks to the introduction of miniaturised and mobile devices, it is possible now to apply another imaging method in the operating theatre - magnetic resonance imaging (MRI). As was shown in the studies of Papa et al., the high sensitivity of the technique (71-100\%), which was previously used mostly for diagnosing breast cancer (especially in high-risk populations), may be additionally used for the evaluation of the radicality of the BCT procedure. The ClearSight ${ }^{\mathrm{TM}}$ system, used by the authors, allows to evaluate ex vivo tissue specimens (after the tumour resection). A short measuring time (1-2 seconds) allows for the verification of the tumour margins, performed in real time. As opposed to standard MRI devices, the system does not require additional shields. Some special attention must be paid to the high value of this technique in differentiating between healthy tissues and cancer infiltration. According to the presented results, the sensitivity of the examination was 91\%, specificity - 93\%, accuracy - 92\% (with the AUC value being 0.97 ). The above measurements were taken on a small group of 22 subjects undergoing BCT. Therefore, the results require confirmation in further studies [32].

A method that has a similarly limited clinical implementation is optical coherence elastography (OCE). During a peri-operative assessment of the resected specimen, the degree of tissue deformation, caused by mechanical pressure, is made. A characteristic property of malignant lesions, used for their differentiation, is their mechanical heterogenicity. The obtained data are presented in a graphic form (elastogram). They also require comparison with the images obtained with the standard tissue staining techniques (haematoxylin and eosin/H\&E/stain) [33, 34].

\section{Fluorescence techniques}

In order to apply this group of diagnostic techniques, it is necessary to use fluorophores (chemical compounds which fluorescence properties induced by light excitation, i.e. by infrared light) and the appropriate equipment [35-38], comprising a source of light with a specific wavelength light-emitting diode (LED), a charge-coupled device (CCD), which works as a detector of the generated radiation as well as a monitor which makes it possible to track the image during the procedure. Apart from the known clinical applications (mapping the lymphatic system in oncological surgery, plastic and reconstructive surgery, cardiac surgery and transplantology) [38], this method has proven to be useful for evaluation of the surgical margins of tumour resection during $\mathrm{BCT}[35,36]$. The most frequently used tracer is indocyanine green (ICG), which has a fluorescence capacity in infrared light with a wavelength of $800-840 \mathrm{~nm}$. As was shown by Kennedy et al., the administration of ICG directly into the cancer lesion and to the subcutaneous tissue lying below it, allows for a credible evaluation of the surgical margins. The sensitivity of the method, as confirmed by the authors was about $70 \%$, whilst specificity was $85 \%$ [36]. 
The introduction of the OST GAINS technique (optical see-through goggle augmented imaging and navigation system) is a continuation of the method based on the use of the LED-CCD devices. Technological progress has made it possible to replace the system registering the effect of the indocyanine green fluorescence (camera, monitor) with the goggles worn by a surgeon. The largest advantage of the new solution is its increased mobility and the fact that the operator is independent from the assistant, whilst the former clinical value of the method is still preserved. In accordance with the suggestion of the authors of the studies carried out with the use of the OST GAINS technique, it is necessary to continue these studies on a larger group of subjects [39].

The use of fluorescence methods is possible also thanks to the more frequent occurrence of overexpression of VEGF-A (vascular endothelial growth factor) in breast cancer patients. This overexpression is diagnosed in a significantly higher rate of breast cancer cases than the overexpression of the HER2 receptor (human epidermal growth factor receptor 2 : in about $73 \%$ and $10-20 \%$ cases respectively). This allows for the possibility of using a complex consisting of an appropriate antibody (bevacizumab) and a fluorescent substance (IRDye800CW) as the tracer. The IRDye800CW fluorescence occurs within the scope of near infrared light with a wavelength of $770-800 \mathrm{~nm}$. In the study carried out by Lamberts et al., which was the first clinical attempt to use the combination of bevacizumab and IRDye800CW on a human model for the purpose discussed here, the efficacy of this method was confirmed. The obtained values of the tracer accumulation level within the tumour, its nearest margins and in the tissue not affected by the tumour were characterised by statistically significant differences $(p<0.05$ and $p<0.0001$, respectively). The tests were carried out on a group of 19 breast cancer patients, 20 assessments of the resected cancer lesions were performed altogether (11 tissue specimens from tumorectomy and 9 tissue specimens after mastectomy were used). However, a limited performance of the technique with regards to the possibility of diagnosing DCIS type lesions within the specimen margins was observed. As the authors suggest, in order to improve the sensitivity, it is necessary to use a larger dose of bevacizumab. Currently it is still not possible with regards to the limitations concerning the use of this antibody for extra-therapeutic purposes established by the FDA (Food and Drug Administration) [40].

\section{Optic techniques}

All diagnostic methods belonging to the group of optic techniques share one property i.e. the necessity to interpret the observed light spectrum (spectroscopy), in structural verifications. In order to evaluate the condition of the tissues which surrounded the resected tumour, the following techniques can be applied: diffuse reflection spectroscopy, fluorescence spectroscopy, optical coherence spectroscopy, bio-impedance spectroscopy, Cerenkov luminescence imaging, Raman spectroscopy (the measurement of Raman radiation scattering - non-elastic photon scattering). Some of the modalities listed here are the methods in which, apart from spectroscopy, some other physical phenomena are applied as well (fluorescence spectroscopy, Cerenkov luminescence).

In order to perform the analysis of diffuse reflectance spectroscopy (DRS), it is necessary to measure light waves with the length of $400-1600 \mathrm{~nm}$. As it is seen in the studies of de Boer et al., this method is valuable in differentiating between normal tissues and cancer infiltration. The authors confirmed the sensitivity of the examination to be $74-79 \%$; specificity - 78-93\% (the AUC value being 0.94). The results were comparable both for the measurements performed in in vivo, and ex vivo conditions, which adds to the value of the test. The lack of necessity to use additional light-sensitive or radioactive substances (as in the fluorescence or isotope methods) is an additional attention-deserving advantage of the test. Some drawbacks of this modality is the effect of the menopausal status of the patient and the type of normal tissues, unaffected by the cancer, on the obtained results [41].

Another clinically available modality is bio-impedance spectroscopy. This technique is based on the variability of dielectric properties of various types of tissues. The practical application of the method is obtained thanks to the ClearEdge device. This examination allows for a high-sensitivity diagnosis of DCIS lesions within the resection margins. Its value does not depend on the structure type of the breast gland (similar results were obtained both for glandular and adipose tissues). According to Dixon et al., the most important advantage of this method is a short learning curve and the possibility of obtaining the final result in a very short time (within 5 minutes, which is identical with the possibility of rendering real-time images) [42]. Also the facility of using the ClearEdge is stressed here, gained thanks to the small size of the device, the cordless system and the availability of the colour-coded image (rendered on an LCD displayer). This technique allows for measurements up to the depth of examined tissues of $3 \mathrm{~mm}$, which sufficiently meets the diagnostic expectations. As it was shown in the clinical studies, the use of this method, decreases the rate of reoperations after $\mathrm{BCT}$, even in as many as $50 \%$ cases. Additionally, this method, as one of the few modalities used for the verification of the resection margins of the primary tumour, allows to evaluate the BCT tumour cavity in a unique manner. The cavity is scanned with a disposable sterile working headpiece of the device [42].

The last of the optical methods discussed in this paper, applies Cerenkov luminescence. This is a type of electromagnetic radiation (in a form of photons), emitted by a dielectric medium, within which the particles with electric charges 
(positrons or electrons) are moving within this medium with the speed higher than the speed of light. The discovery of this type of radiation was awarded in 1958 with the Nobel prize in physics (the awarded team: Pavel Cerenkov, II'ja Mikhailovic, Frank and Igor Yevgenyevich Tamm).

In accordance with the results of the studies carried out by Grootendorst et al., in the methods based on Cerenkov luminescence, it is possible to use tracers, specific for a particular type of tumour (used also in PET — Positron Emission Tomography and SPECT — Single-Photon Emission Computed Tomography), which increases the value of this method [43]. The most frequently used tumour marker is fludeoxyglucose ( $\left.{ }^{18} \mathrm{~F}-\mathrm{FDG}\right)$. The possibility of applying this method in the operating theatre is the outcome of the accessibility of the mobile PET devices (equipment miniaturisation). However, currently, this evaluation of the breast tumour resection margins has some limitations. The time span of the evaluation is still very long. There are also difficulties in obtaining an image with an appropriate resolution (being the outcome of the artefacts resulting from the manipulations of the manually-controlled device and from the respiratory movements of the chest and Gl tract motility) [43].

\section{Isotope methods}

As opposed to the above-listed methods of evaluation of the radicality of primary tumour resection, isotope examinations require the application of ionizing radiation, which is the core problem voiced by the advocates of other methods of verification of the condition of surgical margins.

The marker which is the most frequently used out of all the diagnostic markers in this group is ${ }^{111}$ In or ${ }^{89} \mathrm{Zr}$ radio-labelled bevacizumab $[40,44]$. With regards however to the high requirements concerning the necessary equipment, as well as the necessity to meet the requirements of radiological protection, the attempts carried out to perform this type of evaluation are quite rare and concern limited groups of patients.

\section{Additional surgical procedures to be performed during surgical resection}

In order to minimize the risk of non-radical resection of cancer during $\mathrm{BCT}$, it is possible to perform a routine immediate resection of additional margins from the tumour cavity (cavity shave margins - CSM). In accordance with the recommendations concerning this method, it is necessary to obtain at least four specimens (corresponding to the superior, inferior, lateral and medial margin), each with a minimum width of $1 \mathrm{~cm}$ [45]. In the opinion of Kobbermann et al. it is necessary to resect also the margin on the side of the skin (anterior margin) and on the side of the fascia of the pectoral major muscle (posterior margin) [46]. The above course of treatment is especially helpful in the case of treatment of the breast multi-nodular cancer lesions, undiagnosed preoperatively $[7,46]$. As it was seen from the studies carried out by Bolger et al., in group of patients undergoing $\mathrm{BCT}$, a significantly lower rate of necessary re-operations was observed in those with CSM ( $24 \%$ vs $34 \%$ — in the subjects without CSM) [7]. This observation has also been confirmed by other authors $[47,48]$.

A generally applied method of evaluation of the radicality of the BCT procedure is a gross clinical assessment of the lumpectomy specimen. It is used both for the examination of the tissue specimen and also for the evaluation of the walls of post-surgical cavity, which frequently indicates the

Table I. Alternative possibilities of peri-operative evaluation of the conditions of primary cancer resection margins in breast cancer patients undergoing BCT

\begin{tabular}{|c|c|c|}
\hline $\begin{array}{l}\text { Type of immediate evaluation of } \\
\text { resection margin }\end{array}$ & Advantages of the method & Limitations of the method \\
\hline $\begin{array}{l}\text { Methods of pathological evaluation (touch } \\
\text { imprint cytology, macroscopic evaluation } \\
\text { of resection margins) }\end{array}$ & $\begin{array}{l}\text { - large accessibility } \\
\text { - low cost } \\
\text { - high sensitivity and specificity }\end{array}$ & $\begin{array}{l}\text { - necessity to be evaluated by an experienced } \\
\text { pathologist }\end{array}$ \\
\hline $\begin{array}{l}\text { Radiological methods: } \\
\text { - classical (MMG, USG) }\end{array}$ & $\begin{array}{l}\text { - large accessibility (the standard if imaging } \\
\text { evaluation of the surgical specimen) } \\
\text { - low cost } \\
\text { - procedures with low degree of complexity }\end{array}$ & - limited sensitivity \\
\hline $\begin{array}{l}\text { - the examination of the magnetic } \\
\text { resonance, optical coherence } \\
\text { elastography }\end{array}$ & - high sensitivity and specificity & $\begin{array}{l}\text { - significantly limited accessibility } \\
\text { - complexity } \\
\text { - high cost }\end{array}$ \\
\hline Fluorescence methods & - high sensitivity and specificity & $\begin{array}{l}\text { - significantly limited accessibility } \\
\text { - complexity } \\
\text { - high cost }\end{array}$ \\
\hline Optical methods & - high sensitivity and specificity & $\begin{array}{l}\text { - significantly limited accessibility } \\
\text { - complexity } \\
\text { - high cost }\end{array}$ \\
\hline Isotope method & - high sensitivity and specificity & $\begin{array}{l}\text { - significantly limited accessibility } \\
\text { - complexity } \\
\text { - high cost }\end{array}$ \\
\hline
\end{tabular}


necessity to perform an immediate extension of the scope of primary tumour resection. It seems, however, that in spite of significant advantages (short time and simplicity of the procedure), this type of evaluation of the quality surgical margins is the least efficacious method out of all modalities discussed in this paper [7].

\section{Final remarks}

Peri-operative evaluation of the tissue margins of the surgical specimen after BCT is a definitely required treatment standard [4]. The choice of diagnostic method is a derivative of the equipment and logistic possibilities of oncological centres specialising in breast cancer treatment. The evaluation of the harvested tissues performed solely by the operating surgeon is unacceptable.

Given the economic limitations concerning the principles of operation of healthcare institutions in Poland, it seems justified, in the opinion of the authors, to perform the classical radiological evaluation (MMG, USG) as a priority. An alternative method of verification of radicality of $\mathrm{BCT}$, may be the gross clinical evaluation of the margins performed by a pathologist. The clinical information obtained due to the additional evaluation of the specimen may, at least in some of the cases, protect the patients against the necessity of undergoing another surgery.

\section{Conflict of interest: none declared}

\section{Tomasz Nowikiewicz, MD, PhD}

Oncology Centre — Prof. Franciszek Łukaszczyk Memorial Hospital Department of Clinical Breast Cancer and Reconstructive Surgery

ul. Romanowskiej 2

85-796 Bydgoszcz, Poland

e-mail: tomasz.nowikiewicz@gmail.com

Received: 27 May 2017

Accepted: $4 \mathrm{Jul} 2017$

\section{References}

1. National Comprehensive Cancer Network (2016). National Comprehensive Cancer Network (NCCN). Clinical practice guidelines in oncology: breast cancer version 1.2016. Retrieved 28 March 2016 at: www.ncen.org.

2. Coates AS, Winer EP, Goldhirsch A et al. Tailoring therapies-improving the management of early breast cancer: St Gallen International Expert Consensus on the Primary Therapy of Early Breast Cancer 2015. Ann Oncol 2015; 26: 1533-1546.

3. Buchholz TA, Somerfield MR, Griggs JJ et al. Margins for breast-conserving surgery with whole-breast irradiation in stage I and II invasive breast cancer: American Society of Clinical Oncology endorsement of the Society of Surgical Oncology/American Society for Radiation Oncology consensus guideline. J Clin Oncol 2014; 32: 1502-1506.

4. Piekarski J, Jeziorski A. Leczenie oszczędzające pierś jako pierwotne leczenie bez zastosowania neoadiuwantowego leczenia systemowego. In: Chirurgiczne leczenie zmian nowotworowych piersi: konsensus Polskiego Towarzystwa Chirurgii Onkologicznej. Nowecki Zl, Jeziorski A (eds.). Gdańsk: Via Medica, 2016: 17-19.

5. Jassem J, Krzakowski M (eds.). Rak piersi. In: Zalecenia postępowania diagnostyczno-terapeutycznego w nowotworach złośliwych 2013 rok. Krzakowski M, Warzocha K (eds.). Gdańsk: Via Medica, 2013: 211-263.

6. Herman K, Marczyk E. Rak piersi - rekomendacje Konsultanta Krajowego w dziedzinie chirurgii onkologicznej. In: Rak piersi: postępy diagnostyki i leczenia. Zegarski W, Jastrzębski T, Nowikiewicz T (eds.). Warszawa: Medipage, 2013: 1-6.

7. Bolger JC, Solon JG, Khan SA et al. A comparison of intra-operative margin management techniques in breast-conserving surgery: a standardised approach reduces the likelihood of residual disease without increasing operative time. Breast Cancer 2015; 22: 262-268.

8. NABON. Breast cancer. Dutch Guideline, 2012; http://www.richtlijnendatabase.nl.

9. Morrow M, Harris JR, Schnitt SJ. Surgical margins in lumpectomy for breast cancer — bigger is not better. N Engl J Med 2012; 367: 79-82.

10. Dillon MF, Maguire AA, McDermott EW et al. Needle core biosy characteristics identify patients at risk of compromised margins in breast conservation surgery. Mod Pathol 2008; 21: 39-45.

11. Meijnen P, Oldenburg HS, Peterse JL et al. Clinical outcome after selective treatment of patients diagnosed with ductal carcinoma in situ of the breast. Ann Surg Oncol 2008; 15: 235-243.

12. Dillon MF, McDermott EW, O'Doherty A et al. Factors affecting successful breast conservation for ductal carcinoma in situ. Ann Surg Oncol 2007; 14: $1618-1628$.

13. Jeevan $\mathrm{R}$, Cromwell DA, Trivella $\mathrm{M}$ et al. Reoperation rates after breast conserving surgery for breast cancer among women in England: retrospective study of hospital episode statistics. BMJ 2012; 345: e4505.

14. McCahill LE, Single RM, Aiello Bowles EJ et al. Variability in reexcision following breast conservation surgery. JAMA 2012; 307: 467-475.

15. Kurniawan ED, Wong $M H$, Windle I et al. Predictors of surgical margin status in breast-conserving surgery within a breast screening program. Ann Surg Oncol 2008; 15: 2542-2549.

16. van Deurzen $\mathrm{CH}$. Predictors of surgical margin following breast-conserving surgery: A large population-based cohort study. Ann Surg Oncol 2016; 23 (Suppl 5): 627-633.

17. O'Kelly Priddy CM, Forte VA, Lang JE. The importance of surgical margins in breast cancer. J Surg Oncol 2016; 113: 256-263.

18. Landercasper J, Attai $D$, Atisha $D$ et al. Toolbox to reduce lumpectomy reoperations and improve cosmetic outcome in breast cancer patients: The American Society of Breast Surgeons Consensus Conference. Ann Surg Oncol 2015; 22: 3174-3183.

19. Riedl O, Fitzal F, Mader $\mathrm{N}$ et al. Intraoperative frozen section analysis for breast-conserving therapy in 1016 patients with breast cancer. Eur J Surg Oncol 2009; 35: 264-270.

20. Landercasper J, Whitacre E, Degnim AC et al. Reasons for re-excision after lumpectomy for breast cancer: insight from the American Society of Breast Surgeons Mastery(SM) database. Ann Surg Oncol 2014; 21: 3185-3191.

21. SchwartzT, Degnim AC, Landercasper J. Should re-excision lumpectomy rates be a quality measure in breast-conserving surgery? Ann Surg Oncol 2013; 20: 3180-3183.

22. Morrow M, Katz SJ. The challenge of developing quality measures for breast cancer surgery. JAMA 2012; 307: 509-510.

23. Esbona K, Li Z, Wilke LG. Intraoperative imprint cytology and frozen section pathology for margin assessment in breast conservation surgery: a systematic review. Ann Surg Oncol 2012; 19: 3236-3245.

24. Fleming FJ, Hill AD, Mc Dermott EW et al. Intraoperative margin assessment and re-excision rate in breast conserving surgery. Eur J Surg Oncol 2004; 30: 233-237.

25. Bathla L, Harris A, Davey M et al. High resolution intra-operative two-dimensional specimen mammography and its impact on second operation for re-excision of positive margins at final pathology after breast conservation surgery. Am J Surg 2011; 202: 387-394.

26. Britton PD, Sonoda LI, Yamamoto AK et al. Breast surgical specimen radiographs: how reliable are they? Eur J Radiol 2011; 79: 245-249.

27. Majdak-Paredes EJ, Schaverien MV, Szychta P et al. Intra-operative digital specimen radiology reduces re-operation rates in therapeutic mammaplasty for breast cancer. Breast 2015; 24: 556-559.

28. Edwards SB, Leitman IM, Wengrofsky AJ et al. Identifying factors and techniques to decrease the positive margin rate in partial mastectomies: have we missed the mark? Breast J 2016; 22: 303-309.

29. Unzeitig A, Kobbermann A, Xie XJ et al. Influence of surgical technique on mastectomy and re-excision rates in breast-conserving therapy for cancer. Int J Surg Oncol 2012; 2012: 725121.

30. Thanasitthichai S, Chaiwerawattana A, Phadhana-Anake O. Impact of using intra-operative ultrasound guided breast-conserving surgery on positive margin and re-excision rates in breast cancer cases with current SSO/ASTRO guidelines. Asian Pac J Cancer Prev 2016; 17: 4463-4467.

31. Doyle TE, Factor RE, Ellefson CL et al. High-frequency ultrasound for intraoperative margin assessments in breast conservation surgery: a feasibility study. BMC Cancer 2011; 11: 444. 
32. Papa M, Allweis T, Karni T et al. An intraoperative MRI system for margin assessment in breast conserving surgery: initial results from a novel technique. J Surg Oncol 2016; 114: 22-26.

33. Allen WM, Chin L, Wijesinghe P et al. Wide-field optical coherence micro-elastography for intraoperative assessment of human breast cancer margins. Biomed Opt Express 2016; 7: 4139-4153.

34. Chin L, Latham B, Saunders CM et al. Simplifying the assessment of human breast cancer by mapping a micro-scale heterogeneity index in optical coherence elastography. J Biophotonics 2017; 10: 690-700.

35. Aydogan F, Ozben V, Aytac E et al. Excision of nonpalpable breast cancer with indocyanine green fluorescence-guided occult lesion localization (IFOLL). Breast Care (Basel) 2012; 7: 48-51.

36. Kennedy S, Geradts J, Bydlon T et al. Optical breast cancer margin assessment: an observational study of the effects of tissue heterogeneity on optical contrast. Breast Cancer Res 2010; 12: R91.

37. Murawa D, Hirche C, Dresel $S$ et al. Sentinel lymph node biopsy in breast cancer guided by indocyanine green fluorescence. $\mathrm{Br} J$ Surg 2009; 96: 1289-1294.

38. NowikiewiczT, Kurylcio A, PolkowskiW et al. Imaging methods of the local lymphatic system of the axilla in early breast cancer in patients qualified for the sentinel lymph node biopsy. Prz Menopauz 2016; 15: 52-55.

39. Mondal SB, Gao S, Zhu N et al. Optical see-through cancer vision goggles enable direct patient visualization and real-time fluorescence-guided oncologic surgery. Ann Surg Oncol 2017; 24: 1897-1903.

40. Lamberts LE, Koch $M$, de Jong JS et al. Tumor-specific uptake of fluorescent Bevacizumab-IRDye800CW microdosing in patients with primary breast cancer: A phase I feasibility study. Clin Cancer Res 2017; 23: $2730-2741$.

41. de Boer LL, Hendriks BH, van Duijnhoven F et al. Using DRS during breast conserving surgery: identifying robust optical parameters and influence of inter-patient variation. Biomed Opt Express 2016; 7: 5188-5200.

42. Dixon JM, Renshaw $L$, Young $O$ et al. Intra-operative assessment of excised breast tumour margins using ClearEdge imaging device. Eur $J$ Surg Oncol 2016; 42: 1834-1840.

43. Grootendorst MR, Cariati M, Pinder SE et al. Intraoperative assessment of tumor resection margins in breast-conserving surgery using 18F-FDG Cerenkov luminescence imaging - a first-in-human feasibility study. J Nucl Med 2017; 58: 891-898.

44. Gaykema SB, Brouwers AH, Lub-de Hooge MN et al. 89Zr-bevacizumab PET imaging in primary breast cancer. J Nucl Med 2013; 54: 1014-1018.

45. Hewes JC, Imkampe A, Haji A et al. Importance of routine cavity sampling in breast conservation surgery. Br J Surg 2009; 96: 47-53.

46. Kobbermann A, Unzeitig A, Xie XJ et al. Impact of routine cavity shave margins on breast cancer re-excision rates. Ann Surg Oncol 2011; 18 : 1349-1355.

47. Hequet D, Bricou A, Koual M et al. Systematic cavity shaving: modifications of breast cancer management and long-term local recurrence, a multicentre study. Eur J Surg Oncol 2013; 39: 899-905.

48. Yang $\mathrm{H}$, Jia W, Chen $\mathrm{K}$ et al. Cavity margins and lumpectomy margins for pathological assessment: which is superior in breast-conserving surgery? J Surg Res 2012; 178: 751-757. 